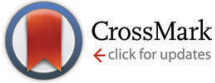

Cite this: J. Mater. Chem. B, 2016, 4, 4104

Received 9th March 2016

Accepted 9th May 2016

DOI: $10.1039 / c 6 t b 00619 a$

www.rsc.org/MaterialsB

\section{Decoupling dual-stimuli responses in patterned lamellar hydrogels as photonic sensors $\dagger$}

\author{
Youfeng Yue, ${ }^{\text {ab }}$ Xufeng Li, ${ }^{a}$ Takayuki Kurokawa, ${ }^{\text {ac }}$ Md. Anamul Haque ${ }^{\text {ad }}$ and \\ Jian Ping Gong*ac
}

\begin{abstract}
This work developed a photonic hydrogel that is responsive to, and can distinguish between two stimuli of stress and $\mathrm{pH}$. Patterning is used to locally change the chemistry of a one-dimensional (1D) photonic gel, such that the native region is responsive to mechanical stress while the chemically modified region is responsive to both mechanical stress and $\mathrm{pH}$. By combining the optical signals in the native region and the modified region, one can distinguish the stimuli between $\mathrm{pH}$ and stress. Specifically, the native 1D photonic gel is composed of periodically aligned polymeric bilayers in a soft polyacrylamide (PAAm) network. The chemical modification is done by partially hydrolyzing PAAm into sodium polyacrylic acid in some patterned regions, which imparts $\mathrm{pH}$ sensitivity, in addition to the stress sensitivity, to these regions.
\end{abstract}

\section{Introduction}

Photonic crystals are materials designed with a periodicity in the dielectric constant, which can affect the propagation of light. The periodic structure of photonic crystals intensifies the reflection at a particular wavelength satisfying the Bragg diffraction and results in brilliant structural colors. Photonic crystals based on soft materials, that is, polymers and gels, have attracted great attention due to their tunable photonic stop-bands under mechanical deformation. ${ }^{1}$ Photonic gels have additional advantages as active chromatic materials through swelling/deswelling in response to environmental stimuli, such as $\mathrm{pH},{ }^{2}$ solvent, ${ }^{3}$ and temperature. ${ }^{4}$ This kind of chromatic stimuli-response behavior in aqueous solution resembles the structural coloration widely found in aquatic animals for camouflage, signaling, and mimicry. ${ }^{5}$

So far, many responsive photonic gels have been developed. For example, 3D photonic gels have been designed by the incorporation of periodically packed colloidal crystal arrays (CCA) into the polymer matrix, or the formation of a porous polymer matrix by etching away the precursor colloidal particles. ${ }^{6}$ Polymerizable amphiphilic molecules that self-assemble into ordered lamellar structures have also been used as a platform for creating responsive photonic gels. ${ }^{7}$ We have developed a 1D photonic hydrogel consisting of lamellar bilayers (polydodecyl glyceryl itaconate:

\footnotetext{
${ }^{a}$ Graduate School of Life Science, and Faculty of Advanced Life Science, Hokkaido University, Sapporo 060-0810, Japan. E-mail: gong@mail.sci.hokudai.ac.jp

${ }^{b}$ Electronics and Photonic Research Institute, National Institute for Advanced Industrial Science and Technology, Tsukuba, 305-8565, Japan

${ }^{c}$ Global Station for Soft Matter, Global Institution for Collaborative Research and Education, Hokkaido University, Sapporo, Japan

${ }^{d}$ Department of Chemistry, University of Dhaka, Dhaka 1000, Bangladesh

$\dagger$ Electronic supplementary information (ESI) available. See DOI: 10.1039/c6tb00619a
}

PDGI) in the polymer matrix of polyacrylamide (PAAm). ${ }^{8}$ The PDGI bilayers (thickness $\sim 5 \mathrm{~nm}$ ) are rigid and non-swellable while the PAAm layers which are several hundred nanometers thick are soft and swellable in water. This photonic hydrogel, referred to as the PDGI/PAAm gel, shows excellent mechanochromic properties. ${ }^{8}$

The photonic hydrogels are promising materials used as intelligent sensors to show a color change in response to one or even multiple environmental stimuli. ${ }^{9}$ However, conventional photonic hydrogels lack the ability to distinguish between multiple stimuli. For example, a porous gel made by copolymerization of $\mathrm{N}$-isopropylacrylamide and methacrylic acid shows a color change due to the change in both temperature and $\mathrm{pH}^{10}$ But it is still difficult from the optical signal to distinguish whether the color change comes from the change in temperature or $\mathrm{pH}$. Besides the lack of selectivity, other properties of photonic crystal based sensors, such as stability, sensitivity, and color quality, are also need to be improved.

In this work, we developed an intelligent photonic gel sensor that is capable of distinguishing between the mechanical stress response and the $\mathrm{pH}$ response. Our strategy is to locally pattern a 1D photonic hydrogel into different chemical regions (Scheme 1). We start from a PDGI/PAAm gel that is stress-sensitive and shows a full color range and a reversible blue shift under compression. ${ }^{8}$ Since this gel shows no response to $\mathrm{pH}$, we partially hydrolyze the PAAm polymer into polyacrylicacid (PAAc) in a certain patterned region. The hydrolyzed region (denoted as PDGI/h-PAAm) becomes pH-sensitive in addition to increased stress sensitivity in comparison to the native region. We can pre-set the same structural color in the virgin region and the hydrolyzed region at free stress and the reference $\mathrm{pH}$, so the patterned gel in this state does not show any pattern. When stress (several $\mathrm{kPa}$ ) is applied or the $\mathrm{pH}$ is changed from the reference value $(\mathrm{pH}=6.9)$, 


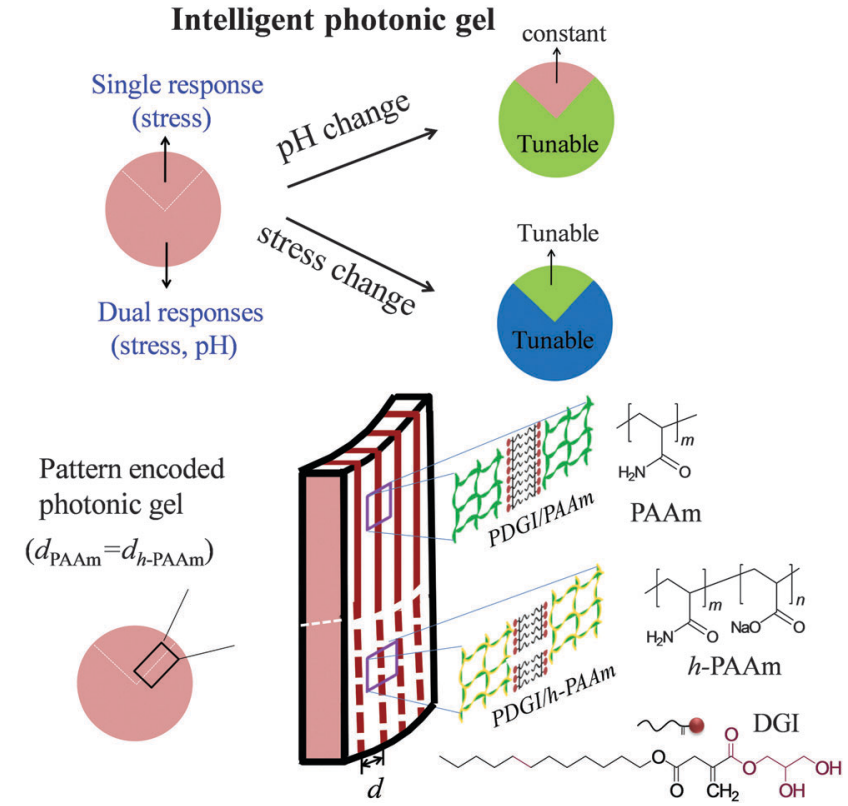

Scheme 1 The native 1D photonic gel, consisting of several thousands of periodically aligned polymeric bilayers (PDGl) in soft gel networks of PAAm, is stress-responsive. The $\mathrm{pH}$ responsibility can be additionally induced by partially hydrolyzing the PAAm polymer into sodium polyacrylic acid, denoted as h-PAAm. By selectively hydrolyzing the gel (bottom), the gel becomes stress responsive in native region (top) and dually responsive to stress and $\mathrm{pH}$ in the hydrolyzed region (bottom). Compared with traditional photonic gel, the proposed intelligent photonic gel can response to, and distinguish $\mathrm{pH}$ and stress.

the pattern appears. By combining the color changes in the native region and the hydrolyzed region, we can distinguish the stimuli between the stress and $\mathrm{pH}$. Since the rigid bilayers embedded in the soft polymer network not only serve as platelets to reflect light, but also as reversible sacrificial bonds to toughen the gel, this dual stimuli-responsive photonic hydrogel can be used repeatedly by displaying and hiding patterns in a fast way. ${ }^{11}$ Such robust photonic films with dual detectability, large signal change, and stability will promote their potential application as multi-functional biosensors mimicking the skin of chameleon that can intelligently sense environmental changes.

\section{Results and discussion}

\section{Preparation of photonic gels and structural characterization}

The PDGI/PAAm gel was synthesized using the method described in the literature. ${ }^{8}$ We hydrolyzed the PDGI/PAAm gel by a controlled hydrolysis reaction in alkali solution. ${ }^{12}$ The detailed procedures of homogenous hydrolysis and the selective patterning process are described in the Experimental section and Fig. S1, ESI. $\dagger$ The cross-section and surface morphologies of the native and hydrolyzed regions were characterized using a scanning electron microscope (SEM) and a 3D violet laser scanning microscope (Fig. 1 and Fig. S2, ESI $\dagger$ ). We found a remarkable difference in polymer structures and surface morphologies between the gel in the native and hydrolyzed regions. In the cross-section of the a)

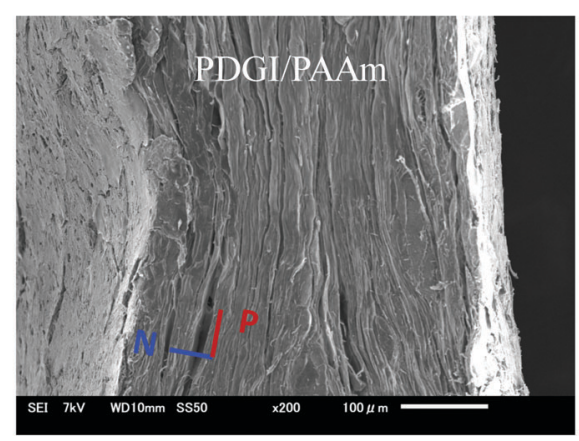

b)

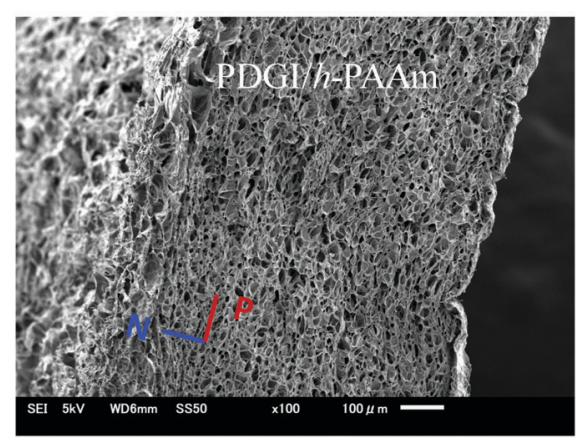

Fig. 1 Cross-sectional SEM images of the photonic gels in the native (a) and hydrolyzed region (b). (N: direction normal to bilayers, P: parallel to the bilayers).

native part, PDGI/PAAm showed a long-range layered structure. In the hydrolyzed region, the bilayer structure was destroyed and many cavities were observed. We will show later that the porous structure favours rapid transport of the fluids into/out of the gel, and improves the response time to the external stimuli.

\section{pH-sensitivity}

First, we studied the pH-response of the chemically modified gel using a homogeneously hydrolyzed sample. Fig. 2a shows the photos and their respective reflection spectra after attaining equilibrium states at various $\mathrm{pH}$ values with a constant ionic strength $(\sim 0.1 \mathrm{M})$. At low $\mathrm{pH}$, the gel (PDGI/h-PAAm) showed color in the ultraviolet region $(\sim 390 \mathrm{~nm})$ and gradually shifted to red $(\sim 600 \mathrm{~nm})$ with increasing $\mathrm{pH}$. The reflection peak wavelength $\left(\lambda_{\max }\right)$ of PDGI/h-PAAm against the $\mathrm{pH}$ of the medium is demonstrated in Fig. 2b. At low $\mathrm{pH}(<3.5), \lambda_{\max }$ was found in the blue-violet wavelength region and increased gradually with $\mathrm{pH}$. At an intermediate $\mathrm{pH}(\sim 3.5-5.0), \lambda_{\max }$ increased sharply, and in the high $\mathrm{pH}$ region $(>5.0) \lambda_{\max }$ saturated to a constant value. While the PDGI/PAAm gel does not respond to $\mathrm{pH}$, there are no acid functional groups in the gel (Fig. 2b).

The red shift of the PDGI/h-PAAm gel, i.e., the increase in $\lambda_{\max }$ with $\mathrm{pH}$, corresponds well to the swelling behaviour of the gel. With the increase in $\mathrm{pH}$, the gel swelled in the thickness direction along with the lateral directions (length and width), as shown in Fig. S3 (ESI $\dagger$ ). The relative swelling in the lateral direction suggests that the bilayer hydrophobic packing is partially destroyed in the lateral direction. ${ }^{11}$ The swelling ratios along the 
a)

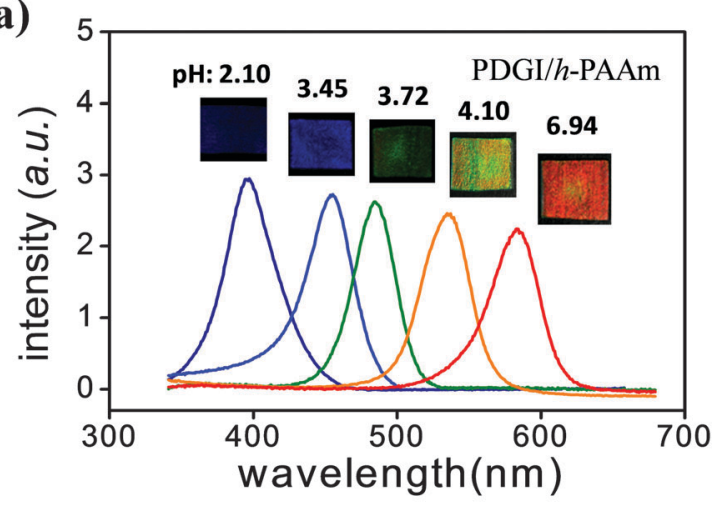

b)

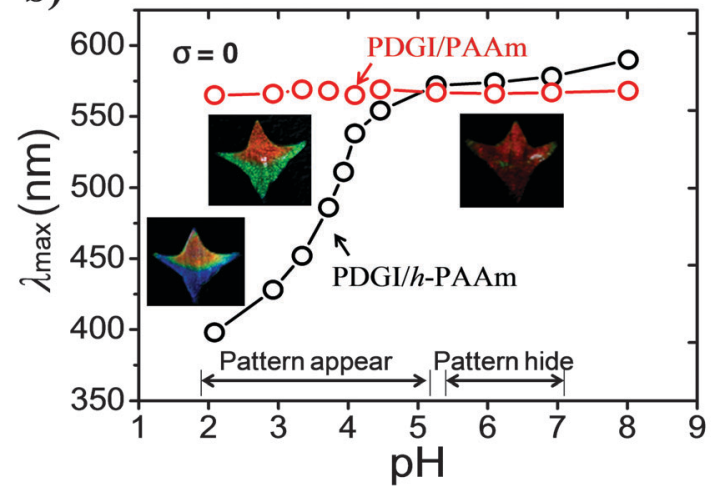

Fig. 2 (a) $\mathrm{pH}$ dependence of the structural colors (inset images) and reflection spectra (Bragg's incident angle $=60^{\circ}$ ) of the PDGI $/ \mathrm{h}$-PAAm gel in the stress-free state $(\sigma=0)$. (b) The $\mathrm{pH}$ dependences of $\lambda_{\max }$ of PDGl/hPAAm and the PDGI/PAAm gel. The former increases gradually with increasing $\mathrm{pH}$, while the latter shows no change with $\mathrm{pH}$. The inset images show color changes of a patterned gel containing native PDGI/PAAm (top region) and PDGI/h-PAAm (bottom region). It can hide and display patterns in different $\mathrm{pH}$ ranges.

bilayers are almost identical to and slightly larger than that perpendicular to the bilayers, which indicates the in-plane isotropic structure and the out-plane anisotropic structure. The dramatic wavelength shift with $\mathrm{pH}$ was due to the swelling of the h-PAAm layer, which increases the periodic layer distance $(d)$. The $d$ can be estimated from $\lambda_{\max }$ by using the Bragg diffraction law, $2 d \sin \theta=\lambda$. The pH response of the PDGI/h-PAAm gel well agrees with the apparent acid dissociation constant $\left(\mathrm{p} K_{\mathrm{a}}=3.5\right)$ of PAAc. ${ }^{13}$ Approximately at $\mathrm{pH}=3$, the hydrolyzed gel starts to swell by proton dissociation with buffer solution going inside the gel. It is interesting to find that the hydrolyzed PDGI/h-PAAm gel showed a much shorter $\lambda_{\text {max }}$ than the native PDGI/PAAm gel in a low $\mathrm{pH}$ range. This indicates that the partially hydrolyzed PAAc groups form hydrogen bonding with PAAm at low $\mathrm{pH}$, which leads to a deswelling of the h-PAAm layer, and therefore a large blue-shift of the PDGI/h-PAAm gel in comparison with the native PDGI/PAAm gel. ${ }^{14}$ This effect dramatically increases the color change sensitivity against the $\mathrm{pH}$ change. So the dramatic swelling with the change in $\mathrm{pH}$ observed in the PDGI/h-PAAm gel results from the combined effect, that is, the destruction of the hydrogen bonding between PAAc and PAAm (enthalpy effect), and the ionic osmotic pressure generated by the dissociation of the carboxyl groups (entropy effect). Although the latter effect is general for charged gels, the former effect is specific for this system.

As the $\lambda_{\max }$ of the PDGI/h-PAAm gel saturated to a value close to that of the native PDGI/PAAm gel at high $\mathrm{pH}$, the selectively hydrolyzed gel displays a pattern at $\mathrm{pH}<5.0$ and hides the pattern at $\mathrm{pH}>5.0$. The fabrication of the patterned gel (PDGI/ PAAm and PDGI/h-PAAm) is demonstrated in Fig. S1 (ESI $\dagger$ ). The inset images of Fig. $2 \mathrm{~b}$ show the color changes with the variation of $\mathrm{pH}$ for a star-like patterned gel selectively hydrolyzed in the bottom region. The native PDGI/PAAm (top region) shows almost the same red color regardless of the change of $\mathrm{pH}$, while the hydrolyzed PDGI/h-PAAm (bottom region) shows a red shift in color from blue, green, to red at $\mathrm{pH}=2.20,4.02$, and 6.96 , respectively. At $\mathrm{pH}>5.0$, the whole gel shows red color and thus the hydrolyzed pattern is hidden. Based on the same method, we can selectively hydrolyze the gel ribbon width " $w$ " or heart-shapes, and these gels also exhibited a selective color variation in pHs (Fig. S4, ESI†).

\section{Response time}

We detected the response time of the PDGI/h-PAAm gel by immersing the water-swollen gels in aqueous solution at different $\mathrm{pH}$ values $(\mathrm{pH}=4.10$ and 3.85). The reflection spectra of the gel were measured with the progress of time (Fig. 3). It can be observed that initially ( $<1 \mathrm{~min}$ ), $\lambda_{\max }$ decreased rapidly and then slowly reached equilibrium states ( $\sim 5 \mathrm{~min})$. The color response time depends on deswelling kinetics. As the swelling/shrinking process of gels is determined by the collective diffusion of the polymer networks in a fluid, the characteristic time is governed by the square of the characteristic size of the gel. ${ }^{15}$ For example, a PAAm-based photonic gel sheet of $\mu \mathrm{m}$-thick has a response time of $\sim 10^{-1} \mathrm{~s}$, whereas the response time of a $1 \mathrm{~mm}$-thick gel sheet becomes $\sim 10^{5} \mathrm{~s}^{16}$ Thus, the observed response time $(\sim 5 \mathrm{~min})$ of the PDGI/h-PAAm gel (size: $10 \times 10 \times 1.7 \mathrm{~mm}^{3}$ ) is much shorter than the value estimated from its thickness $\left(\sim 10^{5} \mathrm{~s}\right)$. The improved response time should be related to the porous structure of the PDGI/h-PAAm gel as shown in Fig. 1b, which favours the rapid transport of the fluids into/out of the gel.

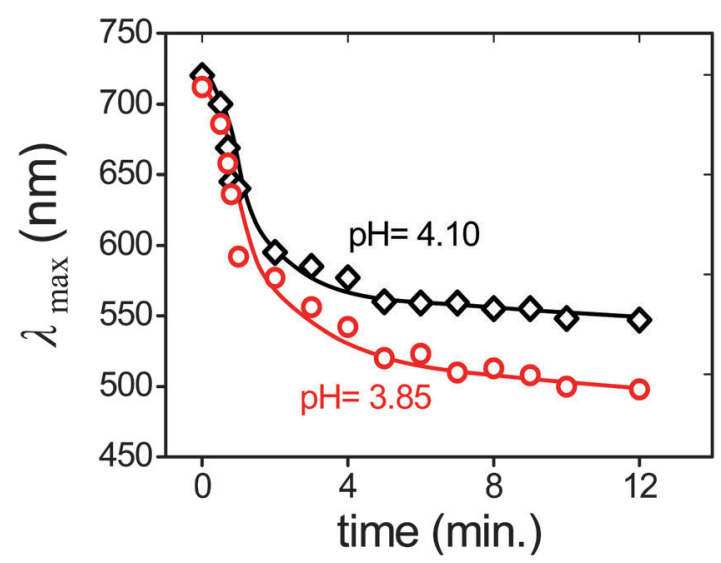

Fig. 3 Response time. Change of the diffraction peak wavelength $\left(\lambda_{\max }\right)$ with progress of time for a water swollen PDGI/h-PAAm gel $(14.5 \times 12.3 \times$ $1.13 \mathrm{~mm}^{3}$ ) immersed in solutions of $\mathrm{pH}=4.10$ and 3.85 . 


\section{Stress sensitivity}

Fig. 4a shows the photos of the PDGI/h-PAAm gel under different compressive stresses $(\sigma)$ in a solution of $\mathrm{pH}=6.94$. The gel was red in the free-standing state $(\sigma=0)$, and changed to blue with a gradual increase in stress. The reflection spectra revealed a blueshift in color upon a gradual increase in stress (Fig. 4b). The PDGI/h-PAAm gel with a porous structure showed a compressive modulus of $\sim 3 \mathrm{kPa}$, much lower than that of the native PDGI/ PAAm gel of $\sim 14 \mathrm{kPa}$ (Fig. S5, ESI $\dagger$ ). So for stress sensors, the PDGI/h-PAAm gel has a higher sensitivity than that of the PDGI/ PAAm gel. As shown in Fig. 4c, for PDGI/h-PAAm at $\mathrm{pH}=6.9$, a compressive stress of $\sim 6 \mathrm{kPa}$ can cause a blue-shift of $\Delta \lambda_{\max }=$ $300 \mathrm{~nm}$, which gives stress sensitivity $\left(\Delta \lambda_{\max } / \sigma\right)$ as high as $\sim 0.05 \mathrm{~nm} \mathrm{~Pa}^{-1}$. For the PDGI/PAAm gel, however, a compressive stress of $\sim 14 \mathrm{kPa}$ causes a blue-shift of $\Delta \lambda_{\max }=185 \mathrm{~nm}$, which gives a stress sensitivity of $\sim 0.013 \mathrm{~nm} \mathrm{~Pa}^{-1}$. These photonic gels have better stress-sensitivities than reported photonic gels, such as electrostatically stabilized colloidal arrays, inverse opal structure and metastable colloidal crystalline arrays. ${ }^{1 a, c, 17}$
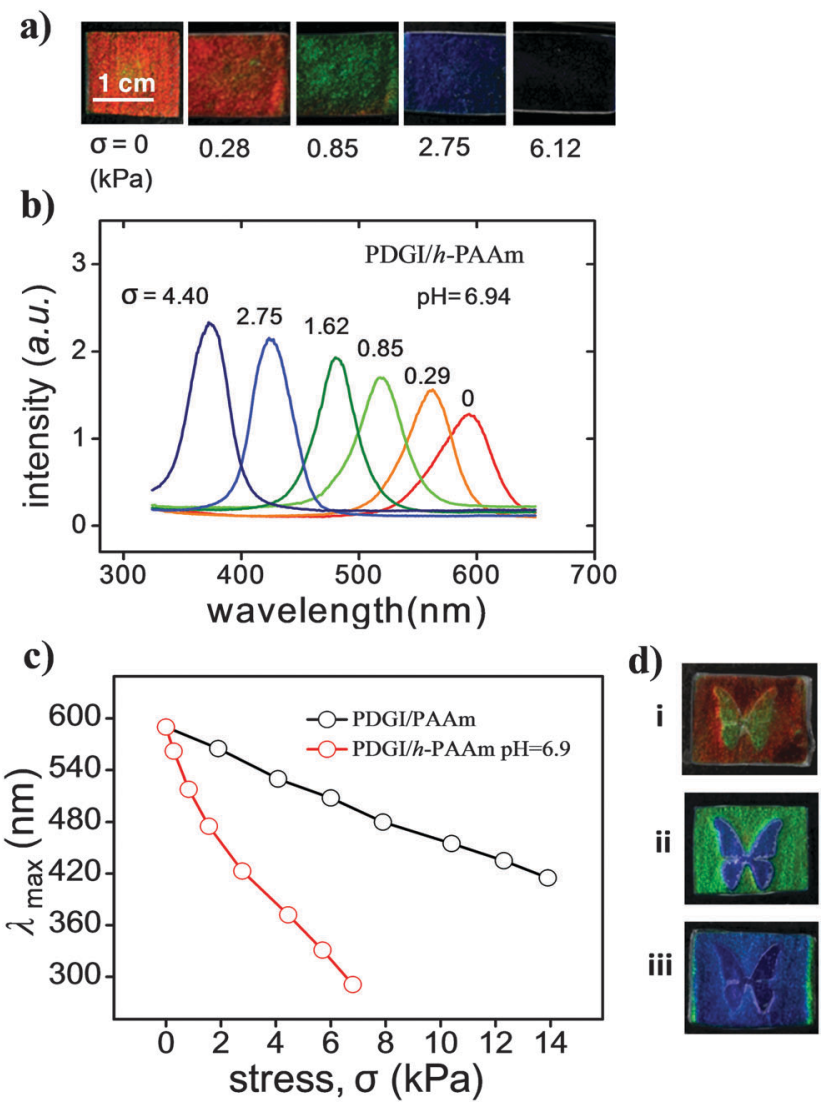

Fig. 4 (a) Photos of the PDGl/h-PAAm gel (swollen in $\mathrm{pH}=6.94$ ) taken at different compressive stresses, $\sigma$. (b) The reflection spectra of gel loaded at different compressive stresses at a constant $\mathrm{pH}$ show shift of $\lambda_{\max }$ from high wavelength (red) to low wavelength region (violet). (c) The $\lambda_{\max }$ decreases with the increasing of compressive stress for PDGI/PAAm gel and PDGl/ h-PAAm gel at $\mathrm{pH}=6.9$. (d) A PDGl/h-PAAm gel exhibits combined response to stress and $\mathrm{pHs}$. The gel swelled in different $\mathrm{pH}$ solutions (i, ii, iii: swollen in $\mathrm{pH}=6.94,4.02,3.51$, respectively) shows different colors. By compressing the gel from top using a piece of glass and a silicone spacer graved with butterfly patterns, the gel shows butterfly patterns of different colors for different $\mathrm{pH}$.
As the color tunability of the PDGI/h-PAAm gel is also dependent on $\mathrm{pH}$, we can show the combined effect of both $\mathrm{pH}$ and stress on the gel. This is demonstrated by compressing the gel swelled in aqueous solution at different $\mathrm{pH}$ values with a piece of glass and a silicone spacer graved with a butterfly pattern. The compressive stress exerted only in the "butterfly" region and the blue-shift of color in this region occurred. For gels swollen in aqueous solution at different $\mathrm{pH}$ values, different colors were observed (Fig. 4d-i-iii). At $\mathrm{pH}=6.94$, the gel was red in background with an orange butterfly pattern (Fig. 4di). At $\mathrm{pH}=4.02$, the background was green and the butterfly was blue (Fig. 4d-ii). At $\mathrm{pH}=3.51$, both the background and the butterfly became blue (Fig. 4d-iii). This result demonstrates that this photonic gel can display different patterns by combining stress and $\mathrm{pH}$.

\section{Dual sensing using the patterned gel}

The above results showed that the native PDGI/PAAm gel only responds to stress while the PDGI/h-PAAm gel can respond to both stress and $\mathrm{pH}$ with a full visible range color change. Therefore, one patterned gel can act as both a pH sensor and two stress sensors with different measuring ranges and sensitivities. Next we demonstrate the dual sensibility using the patterned gel. Fig. 5a illustrates
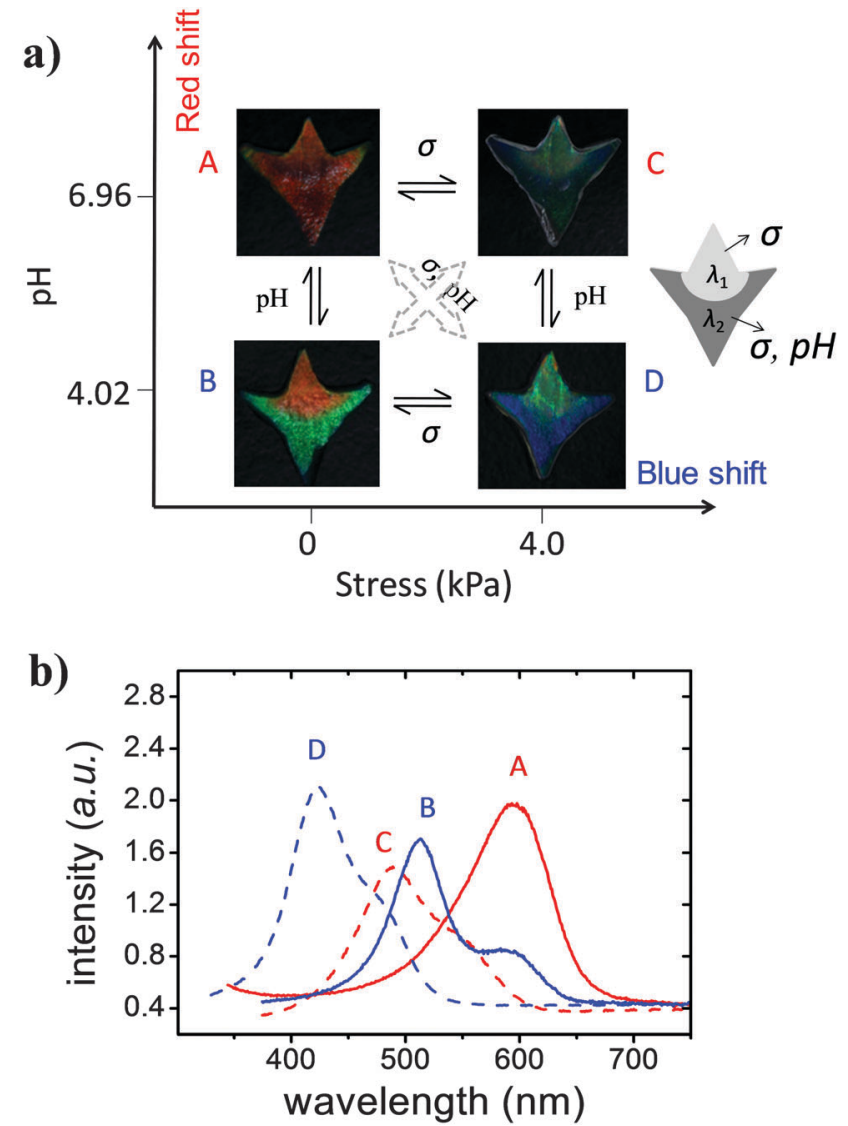

Fig. 5 (a) The upper region of the combined gel is solely response to stress, while the bottom region is response to both $\mathrm{pH}$ and stress. The different states can be reversibily changed and the color information on the top and bottom regions indicates the source of the external stimuli. (b) The reflection spectra of the gel corresponding to the states of A, B, C, D. 
the color tuning of the patterned photonic gel at two $\mathrm{pH}$ values $(\mathrm{pH}=4.02,6.96)$ combined with two compressive stresses $(\sigma=0$, $4.0 \mathrm{kPa}$ ), which gives four different states. The corresponding reflection spectra of the sample are shown in Fig. 5b. Here we denote $\lambda_{1}$ and $\lambda_{2}$ as the spectral peaks of the native and the hydrolyzed regions, respectively. In the A state $(\mathrm{pH}=6.96, \sigma=0)$, the pattern was hidden due to the similar reflection spectra of the native region and the hydrolyzed region $\left(\lambda_{1}=\lambda_{2}\right)$. At $\mathrm{B}(\mathrm{pH}=4.02$, $\sigma=0$ ), only the hydrolyzed region changes color. By using the result in Fig. 2, one can determine the $\mathrm{pH}$ from $\lambda_{2}$. At $\mathrm{C}(\mathrm{pH}=$ $6.96, \sigma=4.0$ ), both of the two regions change color. By using the result in Fig. 4, one can determine the change of stress from $\lambda_{1}$. At $\mathrm{D}(\mathrm{pH}=4.02, \sigma=4.0)$, both $\mathrm{pH}$ and stress change. By using the result in Fig. 4 , one can determine the stress from $\lambda_{1}$. In principle, the $\mathrm{pH}$ in $\mathrm{D}$ can be determined from $\lambda_{2}$ if one knows the $\mathrm{pH}$ dependence of $\lambda_{2}$ at different stresses. So by combining the different color patterns, we can qualitatively identify whether the stimuli come from $\mathrm{pH}$ or stress or from both. All of the four different states are reversible and could be repeated for many times without color degeneration. In addition, the photonic hydrogel stored in water for more than two years did not show any irreversible change in the structure or color, which indicates the excellent stability of the photonic hydrogels.

\section{Conclusions}

In summary, patterned photonic hydrogels with dual-stimuli responses to stress and $\mathrm{pH}$ have been successfully developed. The chemically modified and native regions of the gel can act both as stress sensors with different detection sensitivities, implying that a single gel sensor can detect different scales of stress in a complicated solution environment. The local $\mathrm{pH}$ sensitivity can be used to encode a $\mathrm{pH}$-sensitive pattern in the gel. They can distinguish response to mechanical stresses from the response to $\mathrm{pH}$ with the ease of a visible readout. Such a powerful photonic detector with a combined multi-function is firstly proposed in the photonic crystal based sensors. Another more useful aspect of these hydrogels is that they are biocompatible and robust enough to be cut into very small pieces and could be used as biosensors which could be placed within vessels, for example, to quickly study $\mathrm{pH}$ values of blood in physiological environments or check the blood pressure. We expect that these multi-functional hybrid photonic gels will find many interesting sensor applications.

\section{Materials and methods}

\section{Preparation of the photonic gel}

The parent PDGI/PAAm gel with a lamellar bilayer structure was prepared by simultaneous free radical polymerization from aqueous solution of $0.10 \mathrm{M} 0.027 \mathrm{~mol} \%$ sodium dodecyl sulfate (SDS) relative to DGI, $2 \mathrm{M}$ acrylamide (AAm), $2.5 \mathrm{mM} N, N^{\prime}$ methylenebis(acrylamide) (MBAA) as a cross-linker of AAm, and $2 \mathrm{mM}$ Irgacure as an initiator. ${ }^{8}$ Briefly, prior to polymerization, by applying shear flow to the precursor solution, thousands of lamellar bilayers of self-assembled DGI were aligned in the direction parallel to the substrate. To stabilize these oriented lamellar bilayers, polymerization was performed by irradiating UV light for $8 \mathrm{~h}$ at $50{ }^{\circ} \mathrm{C}$ under an argon gas atmosphere. The polymerization temperature is $50{ }^{\circ} \mathrm{C}$ because the Krafft temperature of DGI is $\sim 43{ }^{\circ} \mathrm{C}$. Below this temperature, it has very low solubility in water. The gel as-prepared is a long strip of $1 \mathrm{~cm}$ width and $0.5 \mathrm{~mm}$ thickness. The prepared gel was immersed in a large amount of water for 1 week to reach an equilibrium swelling state and further cut into different shapes.

\section{Homogeneous hydrolysis of the photonic gel}

Following the previous work, ${ }^{12}$ the PDGI/PAAm gel was hydrolyzed by immersing the gel in $1 \mathrm{M}$ sodium hydroxide aqueous solution for $6 \mathrm{~min}$ at $50{ }^{\circ} \mathrm{C}$. By hydrolysis, the amide groups in PAAm layers were partially converted into carboxylate groups. The obtained sample, denoted as the PDGI/h-PAAm gel, was then extensively washed with deionised water. The PDGI/h-PAAm gel swells largely and changes color from red to far-red due to an increase in the layer distance.

\section{Patterning of the photonic gel}

Selective hydrolysis of the PDGI/PAAm gel was performed by dipping the pre-determined part of the sample in the hydrolysis solution as shown in Fig. S1 (ESI $\dagger$ ).

\section{Acknowledgements}

This study was financially supported by a Grant-in-Aid for Scientific Research (S) (No. 124225006) from Japan Society for the Promotion of Science (JSPS).

\section{Notes and references}

1 (a) A. C. Arsenault, T. J. Clark, G. von Freymann, L. Cademartiri, R. Sapienza, J. Bertolotti, E. Vekris, S. Wong, V. Kitaev and I. Manners, Nat. Mater., 2006, 5, 179-184; (b) S. Ye, Q. Fu and J. Ge, Adv. Funct. Mater., 2014, 24, 6430-6438; (c) D. Yang, S. Ye and J. Ge, Adv. Funct. Mater., 2014, 24, 3197-3205; (d) X. Sun, J. Zhang, X. Lu, X. Fang and H. Peng, Angew. Chem., Int. Ed., 2015, 54, 3630-3634.

2 K. Lee and S. A. Asher, J. Am. Chem. Soc., 2000, 122, 9534-9537. 3 Y. Zhang, Q. Fu and J. Ge, Nat. Commun., 2015, 6, 7510-7516. 4 (a) S. Valkama, H. Kosonen, J. Ruokolainen, T. Haatainen, M. Torkkeli, R. Serimaa, G. ten Brinke and O. Ikkala, Nat. Mater., 2004, 3, 872-876; (b) Y. Ohtsuka, T. Seki and Y. Takeoka, Angew. Chem., Int. Ed., 2015, 127, 15588-15593. 5 S. Kinoshita and S. Yoshioka, ChemPhysChem, 2005, 6, 1442-1459.

6 (a) J. H. Holtz and S. A. Asher, Nature, 1997, 389, 829-832; (b) Y. J. Lee and P. V. Braun, Adv. Mater., 2003, 15, 563-566; (c) I. B. Burgess, L. Mishchenko, B. D. Hatton, M. Kolle, M. Loncar and J. Aizenberg, J. Am. Chem. Soc., 2011, 133, 12430-12432; (d) M. Wang, L. He, W. Xu, X. Wang and Y. Yin, Angew. Chem., Int. Ed., 2015, 54, 7077-7081. 
7 (a) K. Tsujii, M. Hayakawa, T. Onda and T. Tanaka, Macromolecules, 1997, 30, 7397-7402; (b) Y. Yue and J. P. Gong, J. Photochem. Photobiol., C, 2015, 23, 45-67.

8 M. Haque, G. Kamita, T. Kurokawa, K. Tsujii and J. P. Gong, Adv. Mater., 2010, 22, 5110-5114.

9 (a) J. Ge and Y. Yin, Angew. Chem., Int. Ed., 2011, 50, 1492-1522; (b) K. I. MacConaghy, C. I. Geary, J. L. Kaar and M. P. Stoykovich, J. Am. Chem. Soc., 2014, 136, 6896-6899; (c) C. Fenzl, T. Hirsch and O. S. Wolfbeis, Angew. Chem., Int. Ed., 2014, 53, 3318-3335; (d) J. M. Weissman, H. B. Sunkara, S. T. Albert and S. A. Asher, Science, 1996, 274, 959-963; (e) K. Ueno, K. Matsubara, M. Watanabe and Y. Takeoka, Adv. Mater., 2007, 19, 2807-2812; $(f)$ Y. F. Yue, M. A. Haque, T. Kurokawa, T. Nakajima and J. P. Gong, Adv. Mater., 2013, 25, 3106-3110.

10 M. Honda, T. Seki and Y. Takeoka, Adv. Mater., 2009, 21, 1801-1804.
11 Y. Yue, T. Kurokawa, M. A. Haque, T. Nakajima, T. Nonoyama, X. Li, I. Kajiwara and J. P. Gong, Nat. Commun., 2014, 5, 4659-4666.

12 Q. Zhao, J. Sun, Y. Lin and Q. Zhou, React. Funct. Polym., 2010, 70, 602-609.

13 (a) H. Suzuki, B. L. Wang, R. Yoshida and E. Kokufuta, Langmuir, 1999, 15, 4283-4288; (b) M. Honda, T. Seki and Y. Takeoka, Adv. Mater., 2009, 21, 1801-1804.

14 G. Mahdavinia, A. Pourjavadi, H. Hosseinzadeh and M. Zohuriaan, Eur. Polym. J., 2004, 40, 1399-1407.

15 M. J. Lesho and N. F. Sheppard, Polym. Gels Networks, 1998, 5, 503-523.

16 A. Richter, G. Paschew, S. Klatt, J. Lienig, K.-F. Arndt and H.-J. P. Adler, Sensors, 2008, 8, 561-581.

17 S. H. Foulger, P. Jiang, A. Lattam, D. W. Smith, J. Ballato, D. E. Dausch, S. Grego and B. R. Stoner, Adv. Mater., 2003, 15, 685-689. 